\title{
ACTIVIDAD FÍSICA Y GÉNERO: UN ESTUDIO COMPARATIVO ENTRE LOS JÓVENES DE MONTERREY, MÉXICO Y ZARAGOZA, ESPAÑA
}

\author{
Oswaldo Ceballos Gurrola ${ }^{1}$, Javier Álvarez Bermúdez ${ }^{2}$ y Rosa Elena Medina \\ Rodríguez ${ }^{1}$ \\ ${ }^{1}$ Facultad de Organización Deportiva, Universidad Autónoma de Nuevo León, \\ México \\ ${ }^{2}$ Facultad de Psicología, Universidad Autónoma de Nuevo León, México \\ oscegu@hotmail.com
}

\section{RESUMEN}

\begin{abstract}
El propósito del estudio es comparar el gasto energético medio y los niveles de actividad física entre los jóvenes de Monterrey, México y Zaragoza, España; considerando variables como género, tipo de escuela y momento de la semana (jornada escolar o fin de semana). Métodología: estudiantes adolescentes de 12 a 17 años, pertenecientes a las escuelas públicas y privadas de la ciudad de Monterrey $(N=396)$ y Zaragoza $(N=$ 394). Se utilizó el cuestionario Four by one-day physical activity questionnaire con una fiabilidad de .89, realizado con un test-retest por su adaptación de formato. Resultados: existe tanto en Zaragoza como en Monterrey un número importante de escolares con bajos niveles de actividad física. Casi la mitad de los escolares se han tipificado como inactivos y muy inactivos. El gasto energético fue muy similar 37.52 $\mathrm{kcal} / \mathrm{kg} /$ día \pm 2.12 para Zaragoza y $37.66 \mathrm{kcal} / \mathrm{kg} /$ día \pm 2.95 para Monterrey, en las dos ciudades señaladas. Conclusiones: en cuanto al género los hombres presentan mayores niveles de actividad física que las mujeres dato que coincide con la mayoría de los estudios. Los escolares realizan mayor nivel de actividad física durante la jornada escolar que en los fines de semana. Por un lado, obtuvieron un mayor gasto energético los hombres que estudian en las escuelas privadas de Zaragoza y por otro lado, las mujeres de escuelas públicas de Monterrey.
\end{abstract}

PALABRAS CLAVES: adolescentes, gasto energético, niveles de actividad física.

\section{PHYSICAL ACTIVITY AND GENDER: COMPARATIVE STUDY BETWEEN ADOLESCENTS FROM MONTERREY, MEXICO AND ZARAGOZA, SPAIN}

ABSTRACT

\begin{abstract}
The purpose of this study is to compare medium energy expenditure and levels of physical activity between adolescents from Monterrey, Mexico and Zaragoza, Spain taking into consideration variables such as gender, type of school, and time of the week. Methods: adolescent students of 12 to 17 years of age from public and private schools in Monterrey $(N=396)$ and Zaragoza $(N=394) . \quad$ The Four-by-One-Day Physical Activity Questionnaire was used and a .89 reliability with a test-retest because the test was adapted to this study. Results: In Zaragoza as well as in Monterrey there is a significant number of students with low levels of physical activity. Almost half of the students have been classified as inactive and very inactive. The energy expenditure was very similar for both cities: Monterrey $37.52 \pm 2.12 \mathrm{kcal} / \mathrm{kg} /$ day and Zaragoza $37.66 \pm 2.95$ kcal/kg/day. Conclusions: Regarding gender, men show greater levels of physical activity than women. This data coincides with the majority of the studies. Students perform a higher level of physical activity during school days than during weekends.
\end{abstract}

KEY WORDS: adolescents, energy expenditure, levels of physical activity. 


\section{MHSalud}

URL www.una.ac.cr/mhsalud

\section{INTRODUCCIÓN}

La actividad física se define de acuerdo con Caspersen, Powel, y Christenson (1985, p. 126), como "todo movimiento corporal producido por los músculos esqueléticos y que ocasiona un gasto de energía que dependerá de la masa muscular implicada, de las características de dicho movimiento, de la intensidad, duración y frecuencia de las contracciones musculares". Esta definición es una de las más aceptadas y citada en diferentes estudios de actividad física y salud (Kriska, Caspersen y Pereira 1997; Gutiérrez, 2000). Cuando se habla de actividad física se refiere tanto al aspecto deportivo o recreativo, como a todo un conjunto de factores que están presentes en la vida de las personas, como son la experiencia personal, la práctica sociocultural y el movimiento corporal (Devis, 2000).

La actividad física se constituye como un elemento importante en todas las etapas del desarrollo, en la etapa del desarrollo juvenil, la actividad física ofrece grandes posibilidades de mejora de habilidades, hábitos y actitudes que estarán presentes a lo largo de su vida. Son numerosos los trabajos (ACSM, 2009; Álvarez, 2004; Serra, 2001) que han mostrado, como la actividad física regular, produce importantes beneficios en la salud. Pero también se ha encontrado que en muchos adolescentes, la única actividad que realizan en su vida diaria es la que desarrollan durante la clase de educación física (Booth, Okely, Barman \& Macaskill, 2002; Ceballos, Álvarez, Torres y Zaragoza, 2006; Cox, Smith, y Williams, 2008), estando lejos de los estándares mínimos (ACSM, 2009), los cuales son indispensables para obtener beneficios en la salud de las personas.

Los estudios epidemiológicos de la actividad física se han enfocado generalmente a investigar su relación con la salud, enfermedad o conductas como el consumo de alcohol, tabaco, obesidad, etc. El conocimiento de estos estudios es usado en la prevención y control de la enfermedad y la promoción de la salud. Dentro de esta línea, existen estudios epidemiológicos que han señalado los beneficios que proporciona la práctica saludable de una actividad física regular, en el nivel fisiológico, biológico, psicológico y social (Pérez, 2000; Álvarez, 2004), así como sus efectos en diferentes patologías, ya sea en el nivel de prevención o de tratamiento. La práctica de la actividad física casi siempre es beneficiosa, al mejorar el rendimiento de casi todos los sistemas: cardíaco, vascular, respiratorio, músculo esquelético, digestivo, endocrinológico y neurológico (Serra, 2001).

La actividad física ofrece grandes posibilidades para el disfrute y el bienestar psicológico y social, pero por el contrario, posee los riesgos de las lesiones y los derivados de la competitividad y el contexto social que rodea a esta actividad (Devís, 2000). Generalmente, los riesgos son causados por un ejercicio excesivo en cuanto a duración e intensidad. Un ejemplo claro de ello son los deportistas de alto nivel, los cuales están sometidos a riesgos físicos, psicológicos y sociales de diversa índole (Álvarez y López, 2005).

Algunas recomendaciones básicas para la prescripción del ejercicio son que en general el niño, el joven, el adulto, el deportista, etc., aprendan no solo a conocer y dirigir su cuerpo hacia una actividad física determinada, sino también que aprendan a reconocer las dificultades que el espacio físico y social le pueden interponer en la consecución de su actividad, considerando el tipo, intensidad, duración, frecuencia y progresión del ejercicio físico (Álvarez, 2004; Ceballos, 2002). 


\section{MHSalud}

URL www.una.ac.cr/mhsalud

Los métodos de medición que se utilizan en la actividad física y gasto energético son: calorimetría, clasificación del trabajo, marcadores fisiológicos, observación del comportamiento, registros mecánicos y electrónicos (pulsómetros, podómetros y acelerómetros), medidas dietéticas y los métodos de autoinforme (encuestas), siendo estos últimos los instrumentos más prácticos y más ampliamente utilizados para medir la actividad física en el contexto de la investigación epidemiológica en grandes poblaciones (Booth et al., 2002; Kurpad, Raj, Maruthy \& Vaz, 2006; Kriska et al., 1997; Cantera, 1997; Ceballos, Pérez, Medina, Calatayud y Segura, 2005); algunos ejemplos de instrumenos son: cuestionarios de actividad física de recuerdo IPAQ versión corta (Rangul, Holmen, Kurtze, Cuypers \& Midthjell, 2008), 7 -day physical activity diary (Rush, Valencia y Plank, 2008).

Existen muy pocos estudios sobre la actividad física en la población mexicana, uno de ellos es el realizado por la Comisión Nacional del Deporte (CONADE, 2009), que en su informe señala algunas características sobre los hábitos deportivos de los jóvenes, la educación física y la infraestructura. Este estudio plantea la poca calidad y las pocas horas (a veces ninguna) dedicadas por semana a las clases de educación física, el desinterés de los jóvenes de 14 a 18 años que dejan de practicar deporte, pues la materia deja de ser obligatoria en el nivel de bachillerato, así como el incumplimiento de la recomendación dada por la UNESCO respecto de las horas enfocadas al deporte, ya que la cobertura de maestros de educación física está muy por debajo de las necesidades, además de la existencia de instalaciones inadecuadas y sin equipo.

En el caso de Monterrey, México algunos estudios han mostrado que las mujeres practican menos ejercicio que los hombres, ellas lo asocian más a cuerpos esbeltos y distintividad social, en cambio los hombres lo asocian más a ser reconocidos por sus pares, por su voluminosidad muscular y como alternativa a dejar de comer, para estar "bien", practican más deporte en forma colectiva (Álvarez, 2004; Ceballos et al., 2005).

Algunos trabajos respecto al tema de la actividad física presentan una comparación entre poblaciones de distintos países (Dugas et al., 2008; Ceballos et al., 2006) para mostrar las diferencias y similitudes, producto de su propia cultura, lo cual habla de la necesidad de desarrollar estudios de este tipo.

Con base en lo anteriormente expuesto se decide realizar un estudio con el objetivo de comparar el gasto energético medio y niveles de actividad física entre los jóvenes de Monterrey, México y Zaragoza, España considerando variables como el género, tipo de escuela y momento de la semana (jornada escolar o fin de semana).

\section{METODOLOGÍA}

Sujetos: se eligió una muestra al azar con un error del 5\%, de las escuelas secundarias y preparatorias, tanto públicas como privadas de la ciudad de Monterrey como de Zaragoza, una vez obtenidas las escuelas, se eligieron los participantes al azar considerando la proporción por grado y género. La muestra de Monterrey quedó constituida por 396 estudiantes de ambos géneros (193 hombres y 203 mujeres) con edad comprendidas entre los 12 y los 17años, con una media de edad de 14 años; en el caso de Zaragoza, España fue de 394 estudiantes (199 hombres y 195 mujeres) con una media de edad también de 14 años. 


\section{MHSalud \\ URL www.una.ac.cr/mhsalud}

Instrumentos: el cuestionario utilizado para evaluar la actividad física de los estudiantes adolescentes fue el Four by one-day physical activity questionnaire de Cale y Almond (1997) con una adaptación transcultural por Cantera (1997); se considera conveniente, debido a la modificación de formato realizado, constatar la fiabilidad del instrumento aplicando la prueba test-retest; para ello, en una muestra de 20 adolescentes (10 hombres y 10 mujeres) se aplicó el cuestionario de referencia y a las dos semanas el cuestionario modificado; al comparar los resultados, se encontró que el gasto energético medio para el primer cuestionario fue $34^{\prime} 66 \pm 1^{\prime} 62$ mientras que el segundo fue $34^{\prime} 43, \pm 1^{\prime} 70$ estimado en $\mathrm{Kcal} / \mathrm{kg} / \mathrm{día}$.

Para analizar la fiabilidad, en primer lugar se calculó la correlación existente entre los datos mediante la $\rho$ Spearman, siendo esta alta (.89). En segundo lugar, se realizó un contraste de medias mediante la U de Mann-Whitney que resultó no significativo (p>.631), lo que demuestra que no existen diferencias en las medidas obtenidas con ambos cuestionarios. Por todo ello, se puede considerar que el cuestionario modificado es un instrumento fiable y mantiene su estructura original dividiéndose las actividades realizadas por la mañana, tarde y noche; también se tuvo una validación de expertos por la adaptación de contexto cultural (tipo de hábitos de las dos ciudades), teniendo cambios en cuanto al nombre de algunas actividades, en palabras como: piscina-alberca, hacer deberes-hacer la tarea, fregar-trapear, andar-caminar, ordenador-computadora.

Este cuestionario se empleó para conocer el gasto energético medio (G. E. M.) diario en $\mathrm{kcal} / \mathrm{kg} /$ día, según la distribución de las actividades realizadas, agrupadas en categorías de intensidad. Este gasto energético medio será el que registre cada estudiante en diferentes momentos del curso escolar, sin valorar el período vacacional.

El G. E. M. es una de las formas más utilizada en estudios epidemiológicos para medir y clasificar las actividades físicas, esta puede ser en MET o equivalente metabólico. Un MET, es la energía consumida por una persona durante su metabolismo basal o de reposo, que es de aproximadamente $1 \mathrm{Kcal} / \mathrm{kg} /$ hora ó $3.5 \mathrm{ml} / \mathrm{kg} / \mathrm{min}$ (McArdle, Katch \& Katch, 1994; Ainsworth et al., 2000).

Procedimiento: se solicitó el consentimiento tanto de los directivos de las escuelas como de los estudiantes para su participación. El cuestionario se aplicó en el aula en grupos de 10 alumnos con el apoyo de un encuestador. La primera vez que se responde al cuestionario el encuestador comienza leyendo las instrucciones y una a una las preguntas, al mismo tiempo los estudiantes las van contestando, así hasta que se logra comprender totalmente el mecanismo de llevarlo a cabo, para después continuar personalmente.

Análisis estadístico: los datos fueron recogidos en grupos de 10 estudiantes, mediante un cuestionario autoadministrado con el apoyo del encuestador, con la información e instrucciones adecuadas, utilizando un lugar apropiado para su aplicación (sala de clase, de usos múltiples y/o biblioteca).

El análisis estadístico utilizado fue en principio un test de normalidad de las variables (Kolmogorov-Smimov) y posteriormente el contraste de medias (T-Student, $\mathrm{p}<.05$ y IC $95 \%$ ), para comparar el gasto energético según ciudad, grupos de edad, género, tipo de escuela, jornada escolar y fin de semana; los niveles de actividad física se compararon 


\section{MHSalud}

URL www.una.ac.cr/mhsalud

mediante tablas de contingencia con la Chi-cuadrada. Para el procesamiento de los datos se utilizó el programa SPSS versión 15.0.

\section{RESULTADOS}

A continuación se describen los resultados del presente estudio.

Figura 1. Resultados del análisis de T-Test con las variables G. E. M. (kcal/kg/día) por ciudad, edad y género.

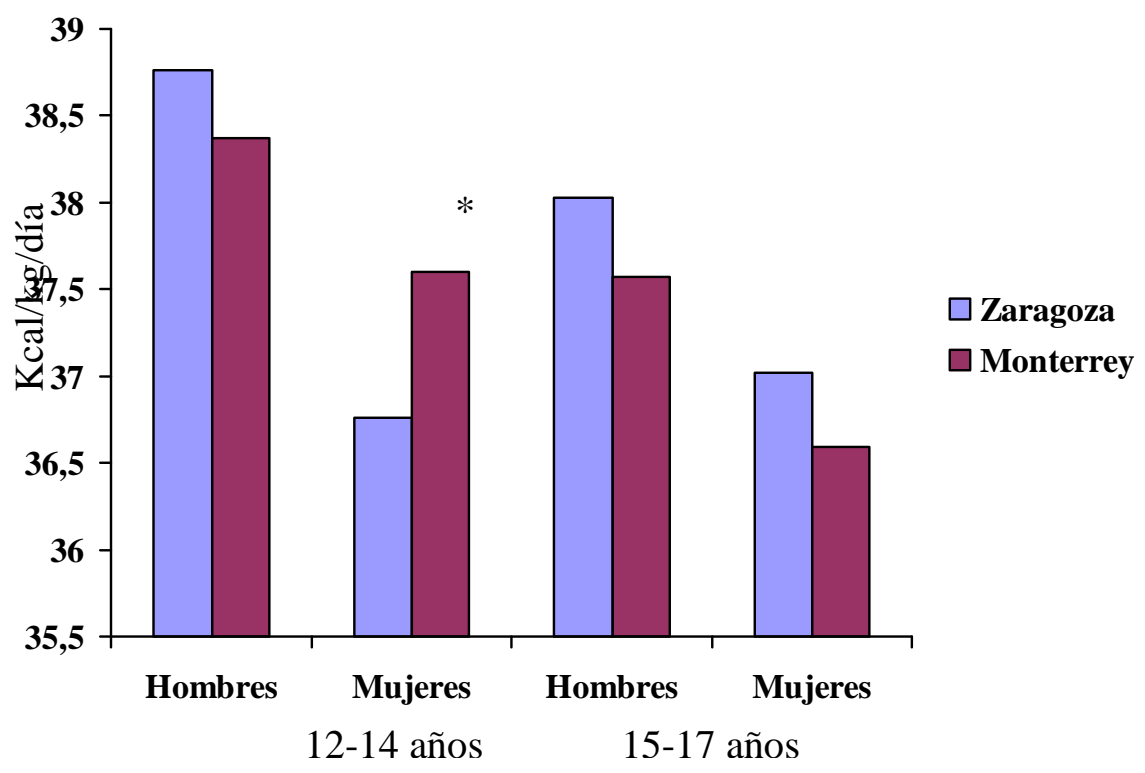

$* \mathrm{p}<.05$

\section{Edad y género}

Al comparar las dos ciudades analizadas en el presente estudio (gráfica 1), no se encuentra diferencias significativas entre el G. E. M. de los hombres de 12-14 años para las dos ciudades estudiadas; sin embargo, las mujeres de Monterrey de este mismo grupo de edad, presentan un gasto superior $(\mathrm{p}=.000), 37.60 \pm 1.96 \mathrm{kcal} / \mathrm{kg} / \mathrm{día}$ frente a Zaragoza $36.76 \pm$ $2.06 \mathrm{kcal} / \mathrm{kg} / \mathrm{día}$. Ahora bien, al comparar tanto a los hombres como a las mujeres de 15-17 años, no se aprecian diferencias significativas entre estas dos ciudades. Es importante señalar que en todo momento los hombres registran ser más activos que las mujeres. 


\section{MHSalud \\ URL www.una.ac.cr/mhsalud}

Figura 2. Resultados del análisis de T-Test con las variables actividades vigorosas

(kcal/kg/día), ciudad, edad y género.

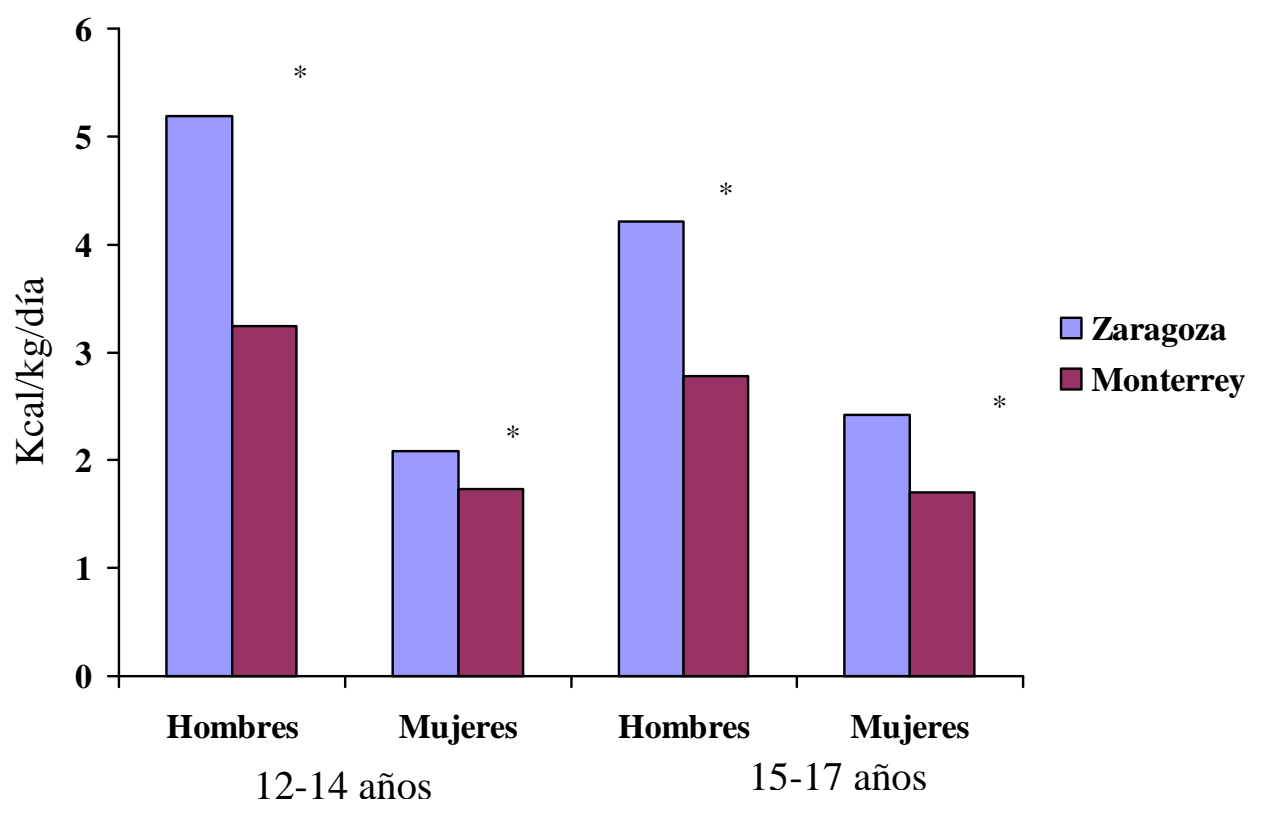

$* \mathrm{p}<0,05$

\section{Actividades vigorosas}

Al analizar el gasto energético medio en actividades vigorosas (actividades clasificadas como fuertes y muy fuertes que ocasionan en la persona un aumento rápido de su frecuencia cardiaca, temperatura corporal y falta de aliento) estimado por los escolares adolescentes de las ciudades de Zaragoza y Monterrey según edad y género, se observa (gráfica 2) que los hombres realizan actividades vigorosas durante más tiempo ( $\mathrm{p}=.003$ ) que las mujeres en las dos ciudades estudiadas $(5.19 \mathrm{kcal} / \mathrm{kg} / \mathrm{día} \pm 3.71 \mathrm{vs} 2.08 \mathrm{kcal} / \mathrm{kg} / \mathrm{día} \pm$ 2.01 y $4.21 \mathrm{kcal} / \mathrm{kg} / \mathrm{día} \pm 3.66$ vs $2.42 \mathrm{kcal} / \mathrm{kg} /$ día \pm 2.65 Zaragoza; $3.24 \mathrm{kcal} / \mathrm{kg} / \mathrm{día} \pm 2.53$ vs $1.73 \mathrm{kcal} / \mathrm{kg} /$ día \pm 1.36 y $2.78 \mathrm{kcal} / \mathrm{kg} /$ día $\pm 2.01 \mathrm{vs} 1.70 \mathrm{kcal} / \mathrm{kg} /$ día \pm 1.11 Monterrey). Además, los escolares de Zaragoza obtienen un gasto energético medio superior en este tipo de actividades respecto a los de Monterrey en cada variable de comparación; dicho de otra manera, los zaragozanos practican más actividades vigorosas y/o deportivas que los regiomontanos. 


\section{MHSalud \\ URL www.una.ac.cr/mhsalud}

Figura 3. Resultados del análisis de T-Test con las variables G. E. M. (kcal/kg/día), ciudad y tipo de escuela.

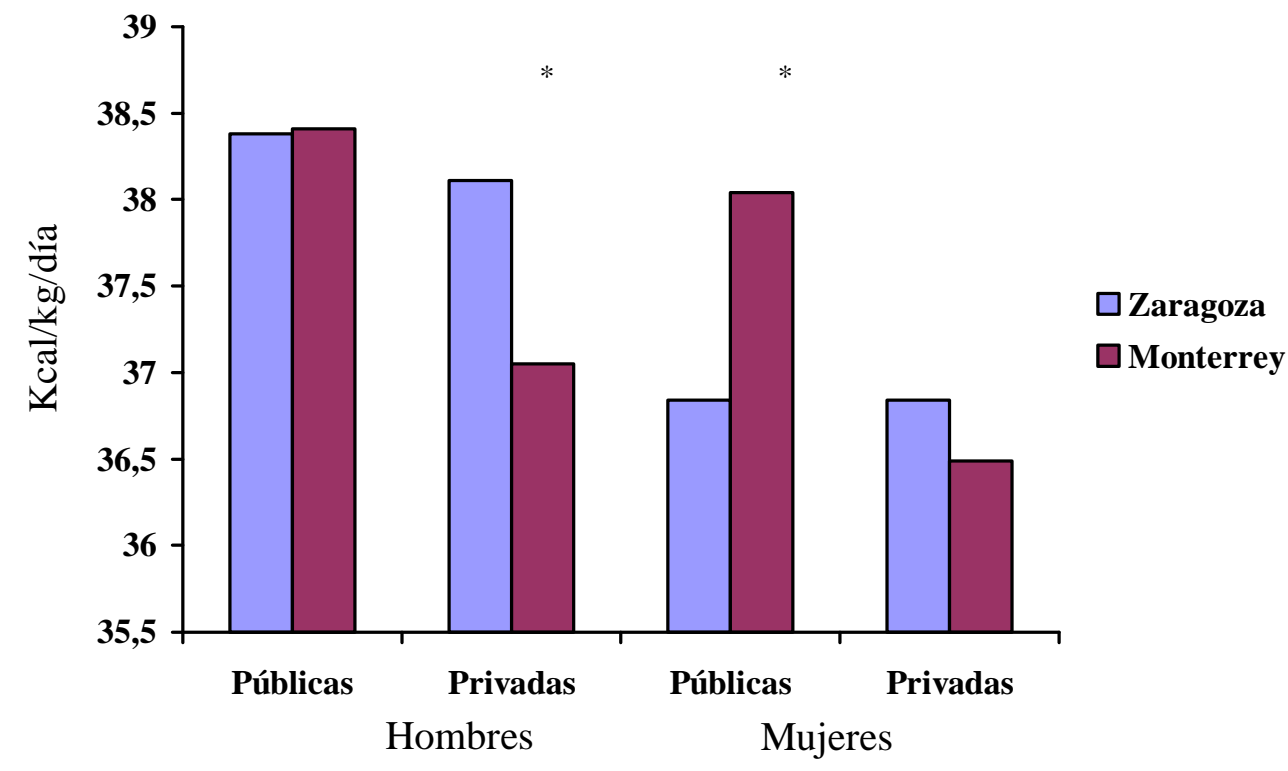

$* \mathrm{p}<.05$

\section{Tipo de escuela}

Al comparar el G. E. M. (kcal/kg/día) de los escolares adolescentes de las ciudades de Zaragoza y Monterrey, se aprecia una diferencia significativa mayor $(p=.015)$ en los hombres que estudian en escuelas privadas de Zaragoza $(38.11 \mathrm{kcal} / \mathrm{kg} / \mathrm{día} \pm 3.01$ vs 37.05 $\mathrm{kcal} / \mathrm{kg} / \mathrm{día} \pm 1.66$ ); por el contrario, las mujeres de escuelas públicas de Monterrey muestran un valor superior (36.84 kcal/kg/día \pm 2.18 vs $38.04 \mathrm{kcal} / \mathrm{kg} / \mathrm{día} \pm 2.02 ; \mathrm{p}=.000)$. 


\section{MHSalud \\ URL www.una.ac.cr/mhsalud}

Figura 4. Resultados del análisis de T-Test con las variables G. E. M. (kcal/kg/día) durante la jornada escolar, por ciudad, edad y género.

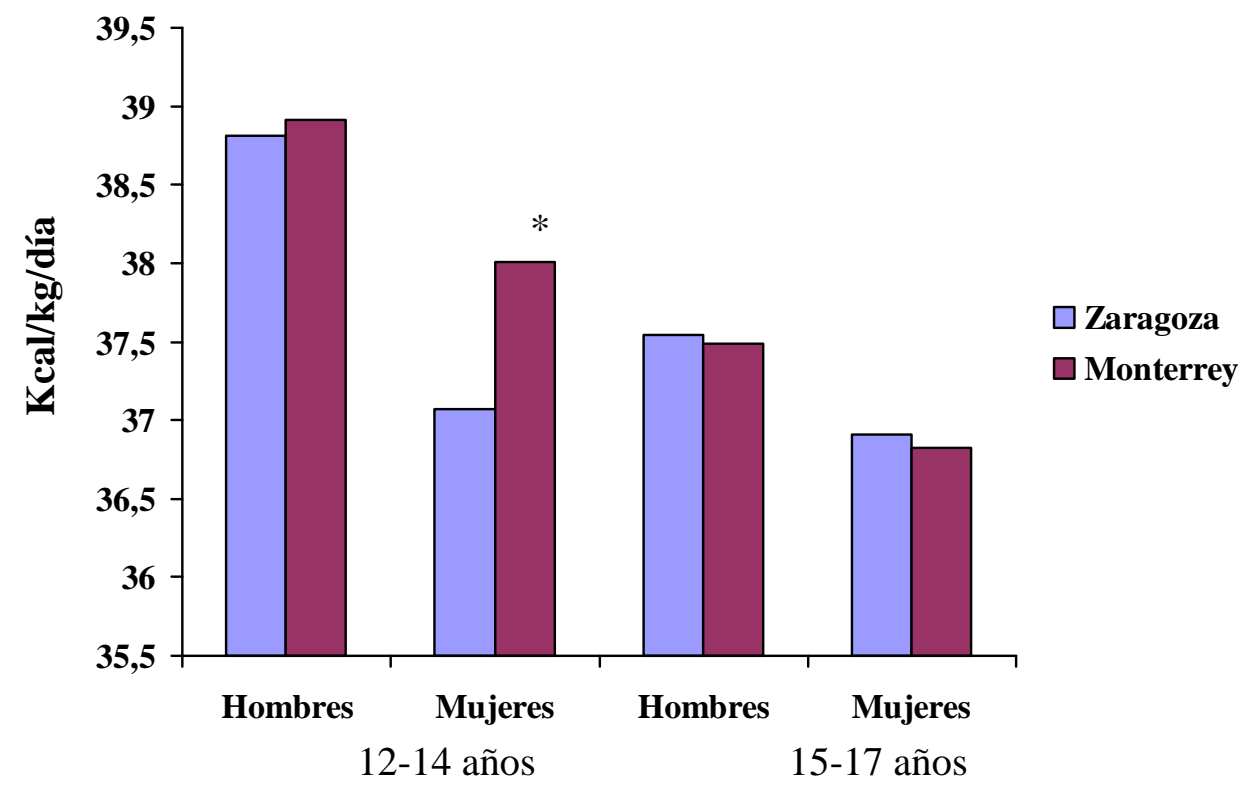

$* \mathrm{p}<0,05$

\section{Jornada escolar y fin de semana}

Sólo las mujeres de 12 y 14 años de la ciudad de Monterrey, presentan un G. E. M. superior $(\mathrm{p}=.026)(38.01 \mathrm{kcal} / \mathrm{kg} / \mathrm{día} \pm 2.45)$ a las de Zaragoza $(37.07 \mathrm{kcal} / \mathrm{kg} / \mathrm{día} \pm 2.48)$, durante la jornada escolar. Sin embargo, para el resto de las comparaciones realizadas por grupos de edad y sexo no se dan diferencias (Gráfica 4).

En la siguiente gráfica (5), se observa que las mujeres de la ciudad de Monterrey (37.20 kcal $/ \mathrm{kg} /$ día \pm 2.36$)$ realizan más actividad física ( $\mathrm{p}=.033)$ durante el fin de semana, respecto a las de Zaragoza (36.46 kcal $/ \mathrm{kg} / \mathrm{día} \pm 2.95)$. En las demás variables comparadas no hay diferencias. 


\section{MHSalud \\ URL www.una.ac.cr/mhsalud}

Figura 5. Resultados del análisis de T-Test con las variables G. E. M. (kcal/kg/día) durante el fin de semana, por ciudad, edad y género

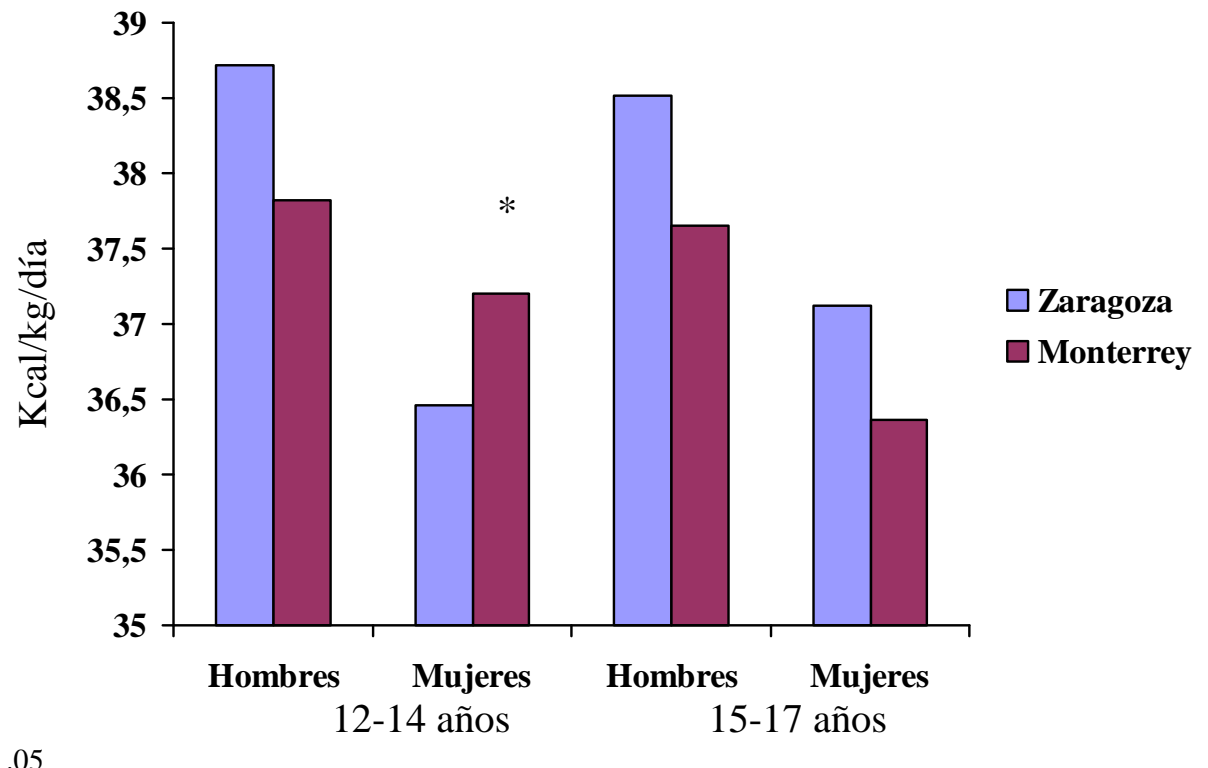

$* \mathrm{p}<.05$

\section{Niveles de actividad física globales}

La comparación de los niveles de actividad física globales de las dos ciudades analizadas, se presenta en la tabla 1; se puede resaltar que la ciudad de Zaragoza tiene un mayor porcentaje de escolares activos (19.3\%) con respecto a Monterrey (10.9\%); por el contrario, existen más escolares moderadamente activos en Monterrey (46.7\%) que en Zaragoza $(33.5 \%)$. 
Tabla 1. 1 Resultados del análisis de Chi-cuadrada con los variables niveles de actividad física y ciudad

\begin{tabular}{|c|c|c|c|c|c|c|}
\hline \multicolumn{2}{|r|}{ Niveles de Actividad Física } & \multicolumn{2}{|c|}{ Zaragoza } & \multicolumn{2}{|c|}{ Monterrey } & \multirow{2}{*}{$\begin{array}{c}\text { Chi- } \\
\text { cuadrada }\end{array}$} \\
\hline & & $\mathrm{n}$ & $\%$ & $\mathrm{n}$ & $\%$ & \\
\hline 1 & Activo (40 o más Kcal/Kg/Día) & 76 & 19.3 & 43 & 10.9 & \\
\hline 2 & Moder. Activo (Entre 37 y 39.99 Kcal/Kg/Día) & 132 & 33.5 & 185 & 46.7 & \\
\hline 3 & Inactivo (Entre 33 y 36.99 Kcal/Kg/Día)Muy & 184 & 46.7 & 164 & 41.4 & $\mathrm{p}=.000$ \\
\hline 4 & Inactivo (Menos de $33 \mathrm{Kcal} / \mathrm{Kg} /$ Día) & 2 & 0.5 & 4 & 1.0 & \\
\hline & Total & 394 & 100 & 396 & 100 & \\
\hline
\end{tabular}

\section{DISCUSIÓN}

Se encontró que existen promedios similares en la variable gasto energético entre las dos muestras de estudio, lo cual de acuerdo a la clasificación de Cale y Almond, (1997) y Cantera (1997) son personas moderadamente activas. Dicho dato permite suponer que, al parecer, existe una disposición cultural de poca estimulación a realizar actividad física (Dugas et al., 2008), ya que en ambas muestras hay un número importante de escolares con bajos niveles de actividad física, donde casi la mitad de los mismos se han tipificado como inactivos y muy inactivos.

Por otro lado, respecto a la variable actividad vigorosa, se encuentran que existen diferencias entre las muestras, los jóvenes mexicanos dedican menos tiempo a las mismas comparados con los de España, lo que habla de que los jóvenes mexicanos en su actividad diaria no realizan actividades que les implique un esfuerzo físico, a diferencia de los españoles que reportan realizar un poco más, pero sin ser óptimo en ambos grupos, ya que lo sugerido sería de al menos 30 minutos diarios de acuerdo a la ACSM (2009), lo anterior se puede deber a que existen factores relevantes que pueden influir en el nivel del gasto energético medio de los jóvenes como son: el tiempo dedicado a ver la televisión, el poco tiempo que se dedica en las escuelas tanto públicas como privadas a la actividad física, así como la cultura personal y familiar.

Asimismo, se encuentra una tendencia de que los hombres realicen actividades físicas durante más tiempo en comparación con las mujeres y por consiguiente obtienen un mayor gasto energético. Al parecer este resultado es una tendencia transcultural de género, ya que resultados de otras investigaciones coinciden en que los hombres son más activos que las mujeres, además éstas tienen niveles más bajos en el gasto energético y que en general las personas disminuyen su actividad física conforme a la edad (Domínguez, Borrell, Nebot \& Plasencia, 1998; Castillo y Balaguer, 1998; Piéron, Telama, Almond \& Barreiro, 1999; Telama \& Yang, 2000; Cantera y Devís, 2000; Tercedor, 2001; Cantera y Devís, 2002; Trost et al., 2002; Ekelund, Yngve, Brage, Westerterp \& Sjöström, 2004; Jago, Baranowski, 


\section{MHSalud}

URL www.una.ac.cr/mhsalud

Zakeri \& Harris, 2005; Bratteby, Sandhagen, Samuelson, 2005; Barnett, Gauvin, Craig \& Katzmarzyk, 2007; Dugas et al., 2008).

En cuanto al gasto energético y tipo de escuela, se ha observado que los escolares de escuelas privadas de la ciudad de Zaragoza son más activos que los de escuelas públicas; por el contrario, los escolares de Monterrey que estudian en escuelas públicas obtienen un mayor gasto energético en contraste con los que estudian en escuelas privadas y además se muestran más activos que los de las escuelas públicas de Zaragoza. Por el contrario en las escuelas privadas se reporta menos práctica de actividad física siendo menor aún en las escuelas privadas de Monterrey. Al parecer en México se da más oportunidad a los jóvenes de desarrollar actividades físicas en el ámbito escolar público, dichos datos nos orientan también a opinar que quizás en las escuelas privadas estudiadas de ambas poblaciones no se le dedica mucho tiempo a la actividad física, quizás por no considerarlo prioritario en la formación de los estudiantes. Asimismo, estos datos difieren de los de otros estudios con muestras españolas, ya que Cantera (1997) no encontró diferencias con respecto a la práctica de actividad física, según el tipo de centro escolar en la provincia de Teruel. Por su parte, Tercedor (1998) tampoco encontró diferencias en las niñas de 10 años, pero sí en los niños de la misma edad, pues realizan más actividad física los de centros privados de la ciudad de Granada. Este resultado hace ver la necesidad de conocer los aspectos básicos culturales de los jóvenes respecto a este tipo de actividades para, por ejemplo, ofertar una variedad de actividades que conecte con los intereses y motivaciones tanto de las mujeres como de los hombres para que de esta forma incrementen su actividad física.

Otro dato encontrado es que los escolares realizan más actividad física durante la jornada escolar que en el fin de semana. Lo que habla de que la cultura de actividad física es más bien impuesta desde el ámbito escolar y no del familiar o personal. Se considera probable que la práctica del deporte organizado en el entorno escolar (actividades extraescolares y clases de Educación Física) y el disponer de mejores o más accesibles instalaciones deportivas, podría justificar esta diferencia. Los datos de Gavarry, Giacomoni, Bernard, Seymat \& Falgairette (2003) en cuanto a que los escolares de Francia son más activos durante la jornada escolar que en fin de semana, concuerdan con los de este estudio.

Un resultado destacado en este estudio es que existe un alto número de escolares adolescentes con bajos niveles de actividad física, lo cual se explica señalando como posibles causas, el tiempo dedicado a ver televisión, la existencia de déficit de instalaciones y programas deportivos para estas edades, más allá del ámbito escolar, aunado a hábitos inadecuados como el fumado y el sedentarismo, y también son causa de ello los factores culturales que no tienden a considerar relevante la actividad física en la educación o en la vida diaria (Álvarez, 2004; Henry, Lightowler \& Al-Hourani, 2004).

\section{CONCLUSIONES}

Aunque no se encontraron diferencias significativas en el gasto energético medio de los escolares de Monterrey y Zaragoza, existe en ambas muestras, un número importante de escolares con bajos niveles de actividad física, donde casi la mitad de ellos, se han tipificado como inactivos y muy inactivos. 


\section{MHSalud \\ URL www.una.ac.cr/mhsalud}

También se encontró que las mujeres de 12-14 años de Monterrey son más activas que las de Zaragoza. Sin embargo, los hombres hacen más actividades vigorosas que las mujeres, y los adolescentes de Zaragoza hacen más actividades vigorosas que en Monterrey.

Los niños de las escuelas privadas de Zaragoza son más activos que en Monterrey, pero las mujeres escuelas públicas de Monterrey son más activas que Zaragoza. Sin embargo, los niños de Monterrey tienen más G. E. M que los de Zaragoza, tanto en jornada escolar como el fin de semana.

Se establece entonces una tendencia de que los hombres presenten mayores niveles de actividad física que las mujeres.

Por último, consideramos que el modelo de abordaje de la actividad física utilizado en el presente estudio para medir el gasto energético medio de los escolares es pertinente por su sencillez y fácil aplicación.

\section{BIBLIOGRAFÍA}

Ainsworth, B. E., Haskell, W. L., Whitt, M. C., Irwin, M. L., Swartz, A. M., Strath, S. J., O’brien, W. L., Bassett, D. R., Schmitz, K. H., Emplaincourt, P. O., Jacobs, D. R. y Leon, A. S. (2000). Compendium of physical activities: an update of activity codes and MET intensities. Medicine \& Science in Sports \& Exercise, 32 (9 suppl): S498S516.

Álvarez, J. y López, M. (2005). El grado de inclusión de la psicología del deporte en la preparación de atletas juveniles mexicanos y los problemas psicológicos más frecuentes de los mismos. En García C. H., Montalvo J., Torres A., Ceballos O. y Álvarez J. "La actividad física y la psicología en el deporte. Publicaciones UANL.

Álvarez, J. (2004). Los jóvenes y sus hábitos de salud. Una investigación psicológica e intervención educativa. México: Trillas.

American College of Sports Medicine. (2009). Guidelines for exercise testing and prescription (8 ed). Baltimore (MD): Lippincott Williams \& Wilkins.

Barnett, T. A., Gauvin, L., Craig, C. L. y Katzmarzyk, P. T. (2007). Modifying effects of sex, age, and education on 22-year trajectory of leisure-time physical activity in a Canadian cohort. Journal of Physical Activity and Health, 4(2), 153-166.

Booth, M. L., Okely, A. D., Barman A. E. y Macaskill, P. (2002). Epidemiology of physical activity participation among New South Wales school students. Australian and New Zealand Journal of Public Health, 26(4), 371-374.

Bratteby, L. E., Sandhagen, B. y Samuelson, G. (2005). Physical activity, energy expenditure and their correlates in two cohorts of Swedish subjects between adolescence and early adulthood. European Journal of Clinical Nutrition, 59(11), 1324-1334.

Cale, L. y Almond, L. (1997). The physical activity levels of English adolescent boys. Physical Education and Sport Pedagogy, 2(1), 74-82.

Cantera, M. A. (1997). Niveles de actividad física en la adolescencia. Estudio realizado en la población escolar de la provincia de Teruel. (Tesis doctoral). Universidad de Zaragoza, España. 


\section{MHSalud}

URL www.una.ac.cr/mhsalud

Cantera, M. A. y Devís, J. (2000). Physical activity levels of secondary school Spanish adolescents. European Journal of Physical Education, 5, 28-44.

Cantera, M. A. y Devís, J. (2002). La promoción de la actividad física relacionada con la salud en el ámbito escolar. Implicaciones y propuestas a partir de un estudio realizado entre adolescentes. Apunts Educación Física y Deportes, 67, 54-62.

Caspersen, C. J., Powel, K. E. y Christenson, G. M. (1985). Physical activity, exercise and physical fitness: definitions and distinctions for health related research. Public Health Report, 100(2), 126-131.

Castillo, I. y Balaguer, I. (1998). Patrones de actividades físicas en niños y adolescentes. Apunts Educación Física y Deportes, 54, 22-29.

Ceballos, O. (2002). Actividad y condición física en escolares adolescentes de la ciudad de Zaragoza (España) y Monterrey (México) (Tesis Doctoral). Universidad de Zaragoza, España.

Ceballos, O., Álvarez, J., Torres, A. y Zaragoza, J. (2006). Actividad física y calidad de vida. México: Publicaciones UANL.

Ceballos, O., Pérez, J. A., Medina, M., Calatayud, J. E. y Segura, J. (2005). Cómo medir la actividad física de los escolares. En García C. H., Montalvo J., Torres A., Ceballos O. y Álvarez J. La actividad física y la psicología en el deporte. México: Publicaciones UANL.

CONADE (2009). Diagnóstico y propuestas para la masificación de la activación física y el deporte de alto rendimiento. México: CONADE.

Cox, A. E., Smith, A. L. y Williams, L. (2008). Change in physical education motivation and physical activity behavior during middle school. Journal of Adolescent Health, 43(5), 506-513.

Devis, J. (2000). Actividad física, deporte y salud. Barcelona: INDE.

Domínguez-Berjón MF, Borrell C, Nebot M, Plasencia A. (1998). La actividad física de ocio y su asociación con variables sociodemográficas y otros comportamientos relacionados con la salud. Gaceta Sanitaria, 12,100-109.

Dugas, L.R., Ebersole, K., Schoeller, D., Yanovski, J. A., Barquera, S., Rivera, J., DurazoArzivu, R. y Luke, A. (2008). Very low levels of energy expenditure among preadolescent Mexican-American girls. International Journal of Pediatric Obesity, $3(2), 123-126$

Ekelund, U., Yngve, A., Brage, S., Westerterp, K. y Sjöström, M. (2004). Body movement and physical activity energy expenditure in children and adolescents: how to adjust for differences in body size and age. American Journal of Clinical Nutrition, 79(5), 851-856.

Gavarry, O., Giacomoni, M., Bernard, T., Seymat, M. y Falgairette, G. (2003). Habitual physical activity in children and adolescents during school and free days. Medicine \& Science in Sports \& Exercise, 35(3), 525-531.

Gutiérrez, M. (2000). Actividad física, estilos de vida y calidad de vida. Revista de Educación Física, 77, 5-16. 


\section{MHSalud}

URL www.una.ac.cr/mhsalud

Henry, C. J., Lightowler, H. J. y Al-Hourani, H. M. (2004). Physical activity and levels of inactivity in adolescent females ages 11-16 years in the United Arab Emirates. American Journal of Human Biology, 16(3), 346-353.

Jago, R., Baranowski, T., Zakeri, I. y Harris, M., (2005). Observed environmental features and the physical activity of adolescent males. American Journal of Preventive Medicine, 29(2), 98-104.

Kriska, A. M. y Caspersen, C. J. (1997). A collection of physical activity questionnaires for health- related research. Medicine \& Science in Sports \& Exercise, 29(6), suplemento, S3-S205.

Kurpad, A.V., Raj, R, Maruthy, K. N. y Vaz, M. (2006). A simple method of measuring total daily energy expenditure and physical activity level from the heart rate in adult men. European Journal of Clinical Nutrition, 60(1), 32-40

McArdle, W., Katch, F., Katch, V. (1994). Essentials of exercise physiology.Pennsylvania: Lea and Febiger.

Pérez, V. (2000). Actividad física, salud y actitudes. Valencia: Edetamia.

Piéron, M., Telama, R., Almond, L. y Carreiro, F. (1999). Estilo de vida de jóvenes europeos: un estudio comparativo". Revista de Educación Física, 76, 5-10.

Rangul, V, Holmen, T. L., Kurtze, N, Cuypers, K. y Midthjell, K. (2008). Reliability and validity of two frequently used self-administered physical activity questionnaires in adolescents. BMC Medical Research Methodology, 8(47).

Rush, E. C., Valencia, M. E. y Plank, L. D. (2008). Validation of a 7-day physical activity diary against doubly-labelled water. Annals of Human Biology, 35(4), 416-421.

Serra, J. R. (2001). Corazón y ejercicio físico en la infancia y adolescencia. España: Masson.

Telama, R. y Yang, X. (2000). Decline of physical activity from youth to young adulthood in Finland. Medicine \& Science in Sports \& Exercise, 32(9), 1617-1622.

Tercedor, P. (2001). Actividad física, condición física y salud. Sevilla: Wanceulen.

Tercedor, P. (1998). Estudio sobre la relación entre actividad física habitual y condición física-salud en una población escolar de diez años de edad. (Tesis Doctoral). Universidad de Granada: España.

Trost, S. G., Pate, R. R., Sallis, J. F., Freedson, P. S., Taylor, W. C., Dowda, M. y Sirard. J. (2002). Age and gender differences in objectively measured physical activity in youth. Medicine \& Science in Sports \& Exercise, 34(2), 350-355.

Fecha de recepción: 03 de noviembre del 2008.

Fecha de aceptación: 25 de agosto del 2009.

Fecha de publicación: 31 de diciembre de 2009. 